Fetal Diagnosis and Therapy
Fetal Diagn Ther 2019;46:12-19

DOI: $10.1159 / 000490723$
Received: October 5, 2017

Accepted after revision: June 6, 2018 Published online: July 25, 2018

\title{
Early Detection of Structural Anomalies in a Primary Care Setting in the Netherlands
}

\author{
Francesca Bardi ${ }^{a}$ Eric Smith ${ }^{b}$ Maja Kuilman ${ }^{b}$ Rosalinde J.M. Snijders ${ }^{a}$ \\ Caterina Maddalena Bilardo ${ }^{a}$ \\ ${ }^{a}$ Fetal Medicine Unit, Department of Obstetrics and Gynecology, University Medical Center Groningen, University of \\ Groningen, Groningen, The Netherlands; b Ultrasound Clinic Bovenmaas, Rotterdam, The Netherlands
}

\section{Keywords}

First-trimester screening $\cdot$ Combined test $\cdot$ Screening for congenital anomalies · First-trimester ultrasound . Structural anomalies

\section{Abstract \\ Objective: This study assessed the percentage and type of congenital anomalies diagnosed at first-trimester ultra- sound (US) scan in a primary care setting without following a standardized protocol for fetal anatomical assessment. Materials and Methods: US scans performed between $11^{+0}$ and $13^{+6}$ weeks of gestation in pregnancies with estimated date of delivery between January 1, 2012 and January 1, 2016 were searched. Data were supplemented with results of 20-week scans and pregnancy outcome. Results: Of all scans, $38.6 \%$ were dating scans and $61.4 \%$ were part of first- trimester screening. Anomalies were diagnosed prenatally in 200 (1.8\%) fetuses; 81 (0.7\%) were chromosomal and 119 $(1.1 \%)$ were structural. Of all prenatally detected anomalies, $27 \%(n=32)$ were detected at first-trimester scan, with a false-positive rate of $0.04 \%$. All cases of anencephaly $(n=4)$, encephalocele $(n=2)$, exomphalos $(n=9)$, megacystis $(n=$ 4 ), and limb reduction ( $n=1$ ) were diagnosed. First-trimester}

detection of gastroschisis and congenital heart defects was 67 and 19\%, respectively. Conclusion: In a primary care setting, global fetal anatomical assessment at first-trimester scan without following a standardized protocol detects about $30 \%$ of all structural anomalies and most of the severe anomalies, with an extremely low false-positive rate. We hypothesize that additional training and use of a systematic protocol would improve early detection of structural anomalies.

(c) 2018 The Author(s)

Published by S. Karger AG, Basel

\section{Introduction}

Prenatal screening has been rapidly and continuously evolving since its introduction in the seventies [1]. Since 2007, pregnant women in the Netherlands can opt for first-trimester screening (FTS) by the combined test between $11^{+0}$ and $13^{+6}$ weeks of pregnancy and the anomaly scan at around 20 weeks. Since 2017, genome-wide cellfree fetal DNA (cffDNA) has become available for all women as first-tier screening from 10 weeks of pregnancy onwards. This has led to a drastic decrease in the uptake of the combined test. As a side effect of this new 
screening strategy, early detection of major structural anomalies has also been affected. First-trimester scans are currently mainly performed for dating the pregnancy at about 9-10 weeks, and in the majority of cases no more scans are performed before the 20-week anomaly scan.

Prior to the introduction of cffDNA, the uptake of the FTS was about $35 \%$, and this has hardly changed with the new screening offer. Conversely, the vast majority of women choose for screening for structural anomalies, with an uptake of $>90 \%$.

Although FTS was primarily offered as screening for aneuploidies, a global, but not systematic survey of fetal anatomy was frequently carried out while attempting to obtain a good nuchal translucency (NT) measurement. Recent studies showed that an early anatomical scan at 12-13 weeks, performed by an experienced sonographer instructed to follow a protocol, can detect over $40 \%$ of all structural anomalies and almost $100 \%$ of the severe ones [2-4]. However, it is not yet known whether a dating scan, performed at 12-13 weeks, would serve in this purpose or if a more detailed ultrasound (US) examination should be recommended. The primary aim of this study was to investigate the percentage and type of congenital anomalies diagnosed during a scan performed for dating of the pregnancy, as opposed to the scan performed as part of the FTS. The study also reports on the percentage and type of anomalies that remain undiagnosed at the first-trimester scan and that are only detected at the 20 -week anomaly scan.

\section{Methods}

This is a retrospective study performed in a primary care US clinic in Rotterdam, The Netherlands. Cases were included if an US scan was performed between $11^{+0}$ and $13^{+6}$ weeks of gestation and if the estimated date of delivery was between January 1, 2012 and January 1, 2016. Pregnancies were excluded from the study if a nonviable pregnancy was seen at the time of the first scan or if in fetuses with a normal first-trimester scan no information on the second-trimester anomaly scan $\left(18^{+0}\right.$ to $22^{+6}$ weeks $)$ was available. If an abnormal marker or an anomaly was diagnosed, pregnancy outcome and postnatal follow-up were searched, and if these were not available, the case was excluded (Fig. 1). In case of suspicion of anomalies, additional information on referrals to a fetal medicine unit was included, genetic investigations and pathology reports were retrieved from the clinic databases, and the national birth registry and the pathology and clinical genetics department of the tertiary care center were consulted. All patient and US data were entered into an electronic database (clinical package Astraia $\mathrm{GmbH}$, Munich, Germany). The retrieved data needed for the study were extracted from the clinical package, anonymized, and subsequently transferred to Microsoft Access. SPSS Statistics ver- sion 23.0 (IBM Corp., Armonk, NY, USA) was used to perform descriptive and comparative statistics. All results were considered statistically significant with $\alpha<0.05$.

\section{First-Trimester Scan}

All women attending the clinic underwent an US scan at $11^{+0}$ to $13^{+6}$ weeks, either in the setting of a dating scan or as part of the nuchal scan in the FTS. The following US systems were used for the examinations: EPIQ-7, IU22, Aloka SSD 3500, Aloka alpha 6, Philips $\operatorname{HD}$ (7, 11, and 15), and ATL HDI 5000 with curved array transducers. Transabdominal and transvaginal scans were performed with 9-1 and 10-3 MHz probes, respectively. US examinations were always started transabdominally and only occasionally the transvaginal route was used owing to technical difficulties, mainly because of a high body mass index. Sonographers were instructed to confirm viability, determine the number of fetuses, and measure crown-rump length in order to accurately establish gestational age. Moreover, the following biometric measurements were performed: biparietal diameter, abdominal circumference, head circumference, and femur length. In women choosing FTS, PAPP-A and free $\beta$-hCG levels were also determined in maternal serum, and NT was measured to calculate the risk of aneuploidy according to the Fetal Medicine Foundation (FMF) algorithm. While waiting for an optimal fetal position for the NT measurement, the sonographer also performed within the time allocated for the FTS a global anatomical survey, but without following a standardized protocol. All FTSs were performed by FMF-certified sonographers, while dating scans were performed with the same US equipment in $36.5 \%$ of cases by FMF-certified and in $63.5 \%$ by non-FMF-certified, but experienced sonographers, similarly instructed to globally investigate the fetal anatomy. The time allocated for the investigations was $20 \mathrm{~min}$ for a dating scan and 30 min for FTS.

\section{Classification of Anomalies}

All fetal anomalies reported in the study were subdivided into different categories depending on the organ system affected: central nervous system (including neural tube defects and brain), facial, respiratory, cardiac, gastrointestinal, abdominal wall, urinary, genital, skeletal, and others. Fetuses with two or more different (i.e., different localized errors in morphogenesis) major anomalies were included in the "multiple congenital anomalies" group [5]. Fetuses with more than one anomaly of different severity were classified based on the most severe anomaly. Also, for fetuses with multiple anomalies, the diagnosis was considered as made prenatally when the most severe anomaly was identified prenatally. Structural anomalies in fetuses with a chromosomal anomaly confirmed by amniocentesis or chorionic villus sampling were excluded. Familiar or nonpathological variants of normal anatomy (e.g., short femur, persistent left superior vena cava) were not considered as structural anomalies. Also, no distinction was made between cystic hygroma and NT $\geq 3.5 \mathrm{~mm}$, as there is an overlap between first-trimester US appearance in excessive nuchal fluid collection. Cardiac defects diagnosed in a referral center owing to presence of one or more US markers (i.e., abnormal ductus venosus Doppler, abnormal heart axis, tricuspid regurgitation) observed at $11^{+0}$ to $13^{+6}$ weeks and prompting referral to a tertiary center were considered as detected during the first trimester. 


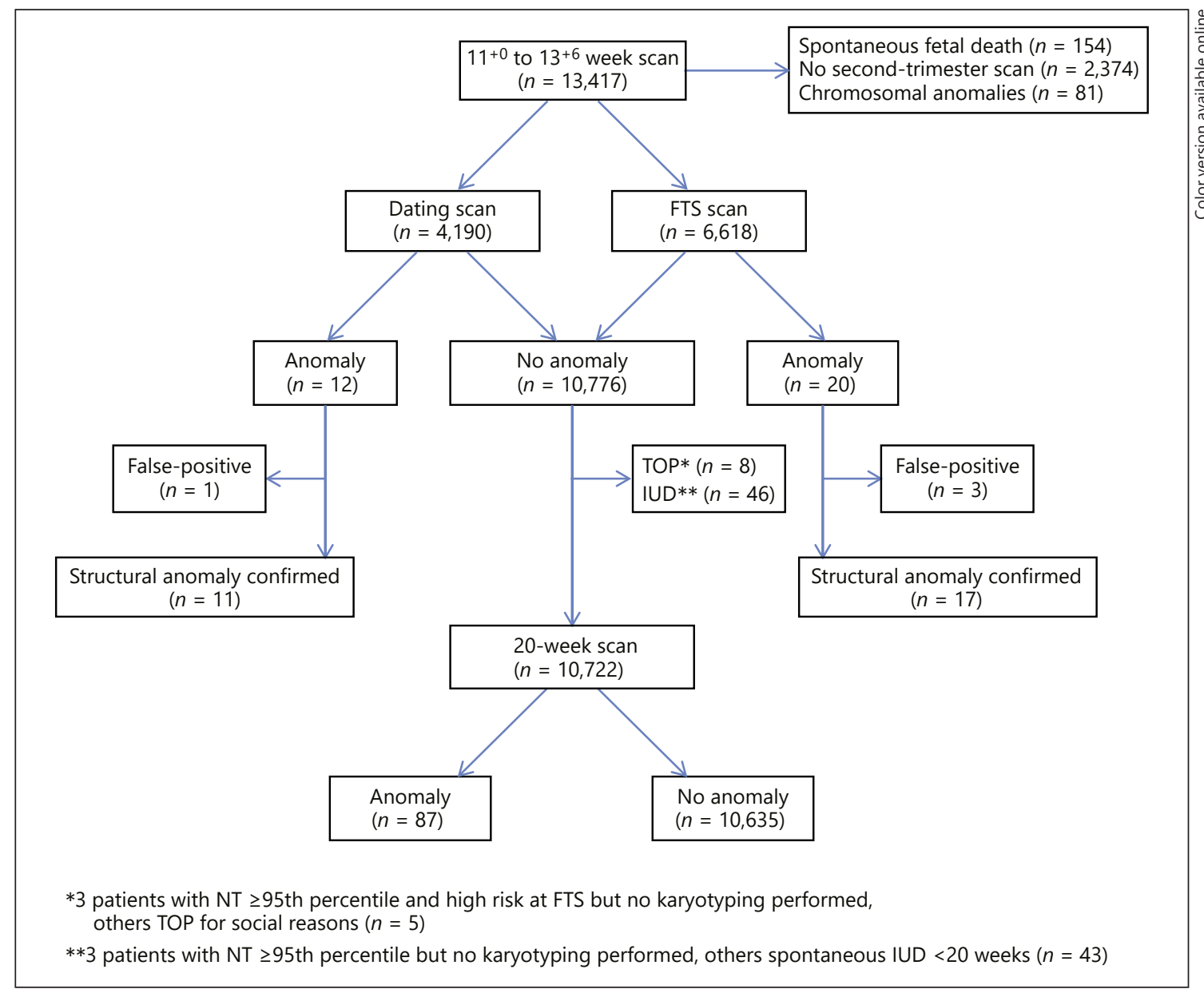

Fig. 1. Flowchart of the patient population. FTS, first-trimester screening; IUD, intrauterine death; NT, nuchal translucency; TOP, termination of pregnancy.

\section{Results}

During the inclusion period, 13,417 pregnancies underwent an US scan between $11^{+0}$ and $13^{+6}$ weeks. First, 154 cases $(1.1 \%)$ were excluded because of fetal demise observed at the time of the first-trimester scan. The pregnancy losses were observed at 11,12 , and 13 weeks in 81 $(52.6 \%), 60(39.0 \%)$, and $13(8.4 \%)$ of the scans performed, respectively. Second, 2,374 (17.7\%) cases were excluded because of missing second-trimester scan data and $81(0.7 \%)$ because of fetal chromosomal anomalies. In total, information on anomalies detected at the first- or second-trimester scan was available in 10,808 pregnancies. Mean maternal age was 30.9 (range 15-46) years and median body weight 67 (range 42-163) kg.

Of all first-trimester scans, 4,190 (38.8\%) were dating scans and 6,618 (61.2\%) FTS scans. The mean gestational age at the time of evaluation was $12^{+1}$ days for dating scans and $12^{+3}$ days for FTS. The prevalence of congenital anomalies in the study population was $1.8 \%(n=200)$, including $81(0.7 \%)$ chromosomal and $119(1.1 \%)$ structural anomalies diagnosed prenatally. First-trimester diagnosis of structural anomalies was $26.9 \%$ (32/119).

\section{Chromosomal Anomalies}

A total of $81(0.7 \%)$ fetuses were diagnosed with a chromosomal anomaly: trisomy $21(n=38,46.9 \%)$, trisomy $18(n=11,13.6 \%)$, trisomy $13(n=6,7.4 \%)$, Turner syndrome $(n=5,6.2 \%)$, triploidy $(n=5,6.2 \%)$, microscopic aberrations $(n=15,18.5 \%)$ detected by microarrays, and 1 case (1.2\%) with abnormal but otherwise unspecified karyotype. The first-trimester diagnosis rate for chromosomal anomalies was $77.7 \%(n=$ 63) (Table 1). 
Table 1. Fetal chromosomal anomalies in the study population

\begin{tabular}{|c|c|c|c|c|c|c|c|}
\hline \multirow[t]{2}{*}{ Chromosomal anomaly } & \multirow[t]{2}{*}{ Total } & \multicolumn{3}{|l|}{ Diagnoses } & \multicolumn{3}{|l|}{ Outcome } \\
\hline & & $11-13$ weeks & $18-22$ weeks & $>22$ weeks & TOP & IUD & live birth \\
\hline Trisomy 21 & $38(46.9)$ & $28(73.7)$ & $8(21.1)$ & $2(5.2)$ & $32(84.2)$ & $1(2.6)$ & $5(13.2)$ \\
\hline Trisomy 18 & $11(13.6)$ & $11(100)$ & - & - & $11(100)$ & - & - \\
\hline Trisomy 13 & $6(7.4)$ & $6(100)$ & - & - & $6(100)$ & - & - \\
\hline Turner syndrome $(45 \mathrm{X} 0)^{\mathrm{a}}$ & $5(6.2)$ & $3(60)$ & - & $2(40)^{\mathrm{a}}$ & $3(60)$ & - & $2(40)^{\mathrm{a}}$ \\
\hline Triploidy & $5(6.2)$ & $5(100)$ & - & - & $4(80)$ & $1(20)$ & - \\
\hline Microscopic aberrations ${ }^{b}$ & $15(18.5)$ & $9(60)$ & $5(33.3)$ & $1(6.7)$ & $4(26.7)$ & $3(20)$ & $8(53.3)$ \\
\hline Unknown & $1(1.2)$ & $1(64.3)$ & - & - & $1(100)$ & - & - \\
\hline Total & 81 & $63(77.7)$ & $13(16)$ & $5(6.2)$ & $59(75.6)$ & $5(6.4)$ & 14 (17.9) \\
\hline
\end{tabular}

Values are presented as $n(\%)$. IUD, intrauterine death; TOP, termination of pregnancy. ${ }^{\mathrm{a}}$ Two patients with mosaic $45 \mathrm{X} 0 .{ }^{\mathrm{b}}$ Including: gain 16p13.11p12.3 $(n=1)$, gain 22q11.2 $(n=2),(1-22) \mathrm{x} 2(\mathrm{XY}) \mathrm{x} 1(n=1)$, 4q del $(n=1)$, LMX1B mutation $(n=1)$, del 22q11.2 $(n=$ 2), del 2q37 $(n=1)$, gain 1q21.1 $(n=1)$, q5 del $(n=1)$, trans 9-13 $(n=1)$, q13 del $(n=1)$, q2 duplication $(n=1)$, 3q2 del $(n=1)$.

\section{Structural Anomalies}

In the study population, a total of 119 (1.1\%) structural anomalies were detected either at the first- or at the second-trimester scan (Table 2). This gives a total prevalence of $1.1 \%$. Of these, $32(26.9 \%)$ were detected in the first trimester. Of the anomalies diagnosed at first-trimester scan, 18 (56.3\%) resulted in termination of pregnancy, $2(6.3 \%)$ in spontaneous fetal demise, and $12(37.5 \%)$ in live births (Table 2).

Eleven out of 12 (91.7\%) abdominal wall defects were diagnosed in the first trimester; omphalocele was detected in all cases (9/9), while gastroschisis was detected in $2 / 3$ cases $(66.7 \%)$.

Multiple congenital anomalies were diagnosed in 3/5 $(60 \%)$ of cases and anomalies of the central nervous system in 6/14 (42.9\%). In the latter group, all cases of acrania $(n=4)$ and encephalocele $(n=2)$ were detected at the first-trimester scan, while all other anomalies, including 3 cases of spina bifida, were overlooked at the early scan.

The overall detection of cardiac anomalies at first-trimester scan was $19.0 \%(4 / 21)$; in 2 cases of tetralogy of Fallot (2/4) and 2 cases of complex heart defects (one with dextrocardia, mitral valve atresia, and hypoplastic aorta, the other with double-outlet right ventricle), first-trimester markers or suspicious findings prompted the referral to a third-level center. All remaining cardiac defects were diagnosed at the 20 -week scan.

Of all skeletal anomalies, $17.6 \%$ (3/17) were diagnosed in the first trimester. Anomalies detected at the early scan included the only case of limb reduction (1/1) as well as $1 / 3$ cases of skeletal dysplasia. All other minor skeletal anomalies, with the exception of $1 / 3$ cases of polydactyly, were diagnosed at the second-trimester scan.

Five out of 33 (15.2\%) urogenital anomalies were diagnosed in the first trimester. This included all cases of megacystis $(n=4)$ and $1 / 5$ cases of unilateral renal agenesis. All other urogenital anomalies, including multicystic renal dysplasia, hydronephrosis, ureterocele, and double collecting kidneys were diagnosed at the 20-weeks scan.

Overall, no cases of gastrointestinal, respiratory, or facial anomalies were detected in the first trimester. These included 3 diaphragmatic hernia, 1 esophageal and $1 \mathrm{du}$ odenal atresia, 5 congenital adenomatoid malformations of the lung, and 7 cases of cleft lip and/or palate.

In the study four false-positive diagnoses were recorded, giving a first-trimester false-positive rate of $0.04 \%$. These included 2 cases of megacystis $<15 \mathrm{~mm}$ that resolved spontaneously and 2 cases of (bowel-only) omphalocele that also resolved spontaneously in the second trimester of pregnancy. All these pregnancies resulted in live births.

\section{Visualization of Fetal Organs at First-Trimester Scan}

Of the 32 anomalies diagnosed in the first trimester, 26 (81.3\%) were detected by FMF-trained sonographers. Although detection was not dissimilar between the two types of investigations, the time reserved for the scan influenced the success of the anatomical survey: completed in $4,030 / 6,618(60.9 \%)$ of the FTS as opposed to in $868 / 4,190(20.7 \%)$ of the dating scans $(p<0.001)$.

Regression analysis was then performed to identify the factors contributing to the successful visualization of fetal 
Table 2. Structural anomalies in the study population and pregnancy outcomes

\begin{tabular}{|c|c|c|c|c|c|c|c|c|}
\hline \multirow[t]{3}{*}{ Fetal anomaly } & \multirow[t]{3}{*}{ Total } & \multicolumn{4}{|l|}{ Diagnoses } & \multicolumn{3}{|c|}{ Pregnancy outcome } \\
\hline & & \multicolumn{3}{|c|}{$11^{+0}$ to $13^{+6}$ weeks $(n=32)$} & \multirow{2}{*}{$\begin{array}{l}20-23 \text { weeks } \\
(\text { FTS/DS) })^{\mathrm{b}}\end{array}$} & \multirow[t]{2}{*}{ TOP } & \multirow[t]{2}{*}{ IUD } & \multirow[t]{2}{*}{ live birth } \\
\hline & & 1 st trimester ${ }^{\mathrm{a}}$ & FTS & DS & & & & \\
\hline Central nervous system & 14 & & & & & & & \\
\hline Acrania/exencephaly & 4 & $4 / 4(100)$ & - & 4 & - & $4(100)$ & - & - \\
\hline Encephalocele & 2 & $2 / 2(100)$ & 2 & - & - & $2(100)$ & - & - \\
\hline Hydrocephaly & 2 & - & - & - & 2 (1 FTS/1 DS) & $1(50)$ & - & $1(50)$ \\
\hline Schizencephaly & 1 & - & - & - & $1(\mathrm{FTS})$ & $1(100)$ & - & - \\
\hline Corpus callosum agenesis & 1 & - & - & - & 1 (FTS) & $1(100)$ & - & - \\
\hline Craniosynostosis & 1 & - & - & - & 1 (FTS) & - & - & $1(100)$ \\
\hline Spina bifida & 3 & - & - & - & 3 (DS) & $2(67)$ & $1(33)$ & - \\
\hline Facial & 7 & & & & & & & \\
\hline Cleft lip & 1 & - & - & - & 1 (FTS) & - & - & $1(100)$ \\
\hline Cleft palate & 1 & - & - & - & $1(\mathrm{DS})$ & - & - & $1(100)$ \\
\hline Cleft lip and palate & 5 & - & - & - & 5 (4 FTS/1 DS) & - & - & $5(100)$ \\
\hline Respiratory & 8 & & & & & & & \\
\hline CAML & 5 & - & - & - & 5 (3 FTS/2 DS) & - & - & $5(100)$ \\
\hline Hernia diaphragmatica & 3 & - & - & - & 3 (FTS) & $1(33)$ & - & $2(67)$ \\
\hline Cardiac & 21 & & & & & & & \\
\hline Double-outlet right ventricle & 1 & - & - & - & 1 (FTS) & - & - & $1(100)$ \\
\hline Transposition great arteries & 2 & - & - & - & 2 (FTS) & - & - & $2(100)$ \\
\hline Coarctation of the aorta & 1 & - & - & - & 1 (FTS) & - & - & $1(100)$ \\
\hline Extended aortic arch & 1 & - & - & - & $1(\mathrm{DS})$ & - & - & $1(100)$ \\
\hline Tetralogy of Fallot & 4 & $2 / 4(50)$ & 2 & - & 2 (1 FTS/1 DS) & $2(50)$ & - & $2(50)$ \\
\hline Hypoplastic left heart & 1 & - & - & - & $1(\mathrm{DS})$ & $1(100)$ & - & - \\
\hline Atrial septal defect & 1 & - & - & - & 1 (DS) & - & - & $1(100)$ \\
\hline Atrioventricular septal defect & 2 & - & - & - & 2 (1 FTS/1 DS) & - & $1(50)$ & $1(50)$ \\
\hline Cor triatriatum dexter & 1 & - & - & - & $1(\mathrm{FTS})$ & - & - & $1(100)$ \\
\hline Aortic valve stenosis & 1 & - & - & - & 1 (FTS) & $1(100)$ & - & - \\
\hline Tricuspid insufficiency & 1 & - & - & - & 1 (FTS) & - & - & $1(100)$ \\
\hline Aortic aneurysm & 1 & - & - & - & 1 (FTS) & - & - & $1(100)$ \\
\hline Right aortic arch & 1 & - & - & - & 1 (FTS) & - & - & $1(100)$ \\
\hline Complex heart defect ${ }^{c}$ & 3 & $2 / 3$ & 2 & - & 1 (FTS) & $2(67)$ & $1(33)$ & - \\
\hline Gastrointestinal & 2 & & & & & & & \\
\hline Esophageal atresia & 1 & - & - & - & 1 (FTS) & - & - & $1(100)$ \\
\hline Duodenal atresia & 1 & - & - & - & 1 (FTS) & - & $1(100)$ & - \\
\hline Abdominal wall & 12 & & & & & & & \\
\hline Gastroschisis & 3 & $2 / 3(67)$ & 2 & - & 1 (FTS) & - & $1(33)$ & $2(67)$ \\
\hline Omphalocele $^{\mathrm{d}}$ & 9 & $9 / 9(100)$ & 4 & 5 & - & $4(44.4)$ & - & $5(55.6)$ \\
\hline Urogenital & 33 & & & & & & & \\
\hline Unilateral renal agenesis & 5 & $1 / 5(20)$ & 1 & - & 4 (4 FTS/1 DS) & $1(20)$ & - & $4(80)$ \\
\hline Megacystis & $4^{\mathrm{d}}$ & $4 / 4(100)$ & 2 & 2 & - & $2(50)$ & - & $2(50)$ \\
\hline Unilateral multicystic renal dysplasia & 8 & - & - & - & 8 (5 FTS/3 DS) & - & - & $8(100)$ \\
\hline Bilateral multicystic renal dysplasia & 2 & - & - & - & 2 (1 FTS/1 DS) & $2(100)$ & - & - \\
\hline Unilateral hydronephrosis & 7 & - & - & - & 7 (5 FTS/2 DS) & - & - & $7(100)$ \\
\hline Bilateral hydronephrosis & 4 & - & - & - & 4 (3 FTS/1 DS) & $1(25)$ & - & $3(75)$ \\
\hline Unilateral ureterocele & 1 & - & - & - & 1 (FTS) & - & - & $1(100)$ \\
\hline Double collecting system & 2 & - & - & - & 2 (FTS) & - & - & $2(100)$ \\
\hline Skeletal & 17 & & & & & & & \\
\hline Limb reduction & 1 & $1 / 1(100)$ & 1 & - & - & - & - & $1(100)$ \\
\hline Polydactyly & 3 & $1 / 3(33)$ & 1 & - & 2 (FTS) & - & - & $3(100)$ \\
\hline
\end{tabular}


Table 2 (continued)

\begin{tabular}{|c|c|c|c|c|c|c|c|c|}
\hline \multirow[t]{3}{*}{ Fetal anomaly } & \multirow[t]{3}{*}{ Total } & \multicolumn{4}{|l|}{ Diagnoses } & \multicolumn{3}{|c|}{ Pregnancy outcome } \\
\hline & & \multicolumn{3}{|c|}{$11^{+0}$ to $13^{+6}$ weeks $(n=32)$} & \multirow{2}{*}{$\begin{array}{l}\text { 20-23 weeks } \\
(\text { FTS/DS })^{b}\end{array}$} & \multirow[t]{2}{*}{ TOP } & \multirow[t]{2}{*}{ IUD } & \multirow[t]{2}{*}{ live birth } \\
\hline & & 1st trimester ${ }^{\mathrm{a}}$ & FTS & DS & & & & \\
\hline Syndactyly and oligodactyly & 1 & - & - & - & 1 (FTS) & - & - & $1(100)$ \\
\hline Club foot unilateral & 2 & - & - & - & 2 (FTS) & - & - & $2(100)$ \\
\hline Club feet bilateral & 6 & - & - & - & 6 (FTS) & - & - & $6(100)$ \\
\hline Rocker bottom feet & 1 & - & - & - & 1 (FTS) & - & - & $1(100)$ \\
\hline Skeletal dysplasia & 3 & $1 / 3(33)$ & 1 & - & 2 (FTS) & $1(33)$ & - & $2(67)$ \\
\hline \multicolumn{9}{|l|}{ Others } \\
\hline Multiple congenital anomalies ${ }^{\mathrm{e}}$ & 5 & $3 / 5(60)$ & 2 & 1 & 2 (FTS) & $3(60)$ & - & $2(40)$ \\
\hline Total & 119 & $32(27)$ & 20 & 12 & 87 & $32(27)$ & $5(4)$ & 82 (69) \\
\hline
\end{tabular}

Values are presented as $n(\%)$. CAML, congenital adenomatoid malformation of the lung; DS, dating scan; FTS, first-trimester screening; IUD, intrauterine death; TOP, termination of pregnancy. ${ }^{a}$ Detected in the first trimester. ${ }^{\mathrm{b}}$ Number of fetuses who underwent DS versus FTS. ${ }^{c}$ One case with dextrocardia, hypoplastic aorta, and aplastic mitral valve, 1 case with transposition of the great arteries and double-outlet right ventricle, and 1 case with unspecified multiple heart defects. ${ }^{\mathrm{d}}$ Two cases of megacystis $<15 \mathrm{~mm}$ with spontaneous resolution and 2 cases of bowel-only omphalocele with spontaneous resolution. ${ }^{\mathrm{e}}$ One case with omphalocele, split hand, dimorphic face, and abnormal skull shape; 1 case with omphalocele and missing foot; 1 case with bilateral hydronephrosis, polydactyly, and ventriculomegaly, and CHARGE syndrome postnatally; 1 case with nasal tumor, cleft lip and palate, hypertelorism, flat nose, dilated heart, and atrial septal defect; 1 case with unspecified heart anomaly, increased nuchal translucency, and missing nasal bone (no karyotype done).

organs at first-trimester scan. This revealed that women undergoing FTS - lasting 30 min - had a 5.6 times higher chance of successful visualization of all fetal organs compared to women who underwent a dating scan $(p<0.001)$. Also, an increase of gestational age by 1 week significantly increased the likelihood of visualization of all fetal organs by 1.7 -fold $(p<0.01)$; all organs were successfully visualized in $24 \%$ of scans performed at 11 weeks, $50 \%$ at 12 weeks, and $52 \%$ at 13 weeks of gestation.

\section{Discussion}

This study showed that a global survey of the fetal anatomy between $11^{+0}$ to $13^{+6}$ weeks of gestation can detect about $30 \%$ of all prenatally diagnosed structural anomalies and that especially the severe ones are amenable to early detection. Factors contributing to a successful detection are the gestational age at the moment of the scan, with higher rates at 13 compared to 11 weeks, and the time allocated for the examination, with more successful complete visualization of the fetal anatomy if the scan is part of the FTS rather than a dating scan. Another important factor was the extremely low false-positive rate $(0.04 \%)$ of early US investigation, confirming our previ- ous report [4]. Moreover, all four false-positive cases concerned defects with a great chance of resolution later in pregnancy, and women were therefore counseled accordingly.

The early detection of anomalies in our study is in line with previous reports on low-risk populations, performed without adherence to a strict protocol and showing a detection rate of about 30\% [6]. Detection can double and can even equal that of second-trimester scans if sonographers are specifically trained to investigate the fetal anatomy systematically [6-8].

The study by Syngelaki et al. [2] is to this date the largest study on early detection of structural anomalies at the nuchal scan, with a detection rate of $44 \%$. Based on the literature, the authors suggested that besides the "always detectable" major anomalies, there is an additional $42 \%$ of potentially detectable anomalies, the recognition of which depends on the experience of the sonographer, the quality of the equipment, and maternal characteristics such as body mass index. In our study, all "always detectable" anomalies (anencephaly, large exomphalos, megacystis, and severe limb defects) were indeed diagnosed in the first trimester. Others, such as gastroschisis, proved again to be more challenging for an early diagnosis [4]. 
In our study we compared early detection at $12-13$ weeks with detection at 20 weeks, without including anomalies detected only after birth. We therefore preferred not to use the terminology "detection rate." However, our observed prevalence of structural anomalies $(1.1 \%)$ is similar to that reported by Syngelaki et al. [2], which is reassuring as to the representativeness of our results, in spite of lack of inclusion of postnatal follow-up. Future prospective studies should overcome this limitation.

The low prevalence of cardiac defects in our population $(0.2 \%)$ suggests that some cardiac anomalies (coronary heart disease) probably also remain undetected at the 20 -week scan. Of the prenatally diagnosed coronary heart disease cases, only $19.0 \%$ (4/21) were diagnosed in the first trimester. This is lower than the $26.4 \%$ reported by Syngelaki et al. [2] and the $56 \%$ reported by Grande et al. [3], but higher than in other previous studies $[9,10]$. In a protocol for first-trimester detection of anomalies, the use of a combination of markers such as NT, ductus venosus flow, tricuspid regurgitation, and measurement of the cardiac axis has the potential of significantly increasing detection rates [11-13].

All sonographers involved in our study were experienced, but had different levels of training. Those performing the FTS were FMF-certified and audited yearly, while most of those performing the dating scans had only basic US knowledge.

Retrospective assessment of the images of anomalies missed by non-FMF-trained sonographers ( 3 spina bifida cases and of $4 / 5$ facial clefts) showed that early markers of abnormal development, such as absent intracranial translucency [14] and abnormal maxilla-nasion-mandible angle, were clearly visible [15]. This suggests that further training in recognition of these markers might have increased detection of these cases.

The lack of a systematic assessment of fetal anatomy in our study applies especially to the dating scan group. In the Netherlands, guidelines recommend performance of dating scans no earlier than at $8^{+4}$ weeks and ideally between $10^{+0}$ and $12^{+6}$ weeks. In reality, most dating scans are performed by midwives at around or before 10 weeks of gestation. The only requirement is a correct crownrump length measurement, with no quality control of operator experience and US equipment used. This study therefore reports on an unusual situation, where dating scans were postponed to beyond 11 weeks and performed by experienced sonographers, although not all trained in FTS. This policy was intentionally chosen by the US clinic where the study was performed to enable early screen- ing for structural anomalies even in women not choosing FTS, but valuing early detection of structural defects.

The study did not show significant differences in the number of anomalies detected at dating or FTS scans. However, anomalies detected at dating scans (acrania, omphalocele, and megacystis) were more obvious than those detected at FTS (cardiac anomalies, renal agenesis, and polydactyly). Moreover, the organ visualization rate at FTS was significantly higher than at dating scans (73.6 vs. $24.1 \%, p<0.001$ ).

In line with previous studies $[16,17]$, this study indicates that training and education play a major role in the successful completion of a detailed US examination and that the optimal time for a first-trimester scan is $12-13$ weeks [ 18 , $19]$, when most fetal organs are sufficiently developed [14, $20,21]$, their visualization can be complete in $86 \%$ of cases [22], the majority of spontaneous losses have already occurred (from 81 at 11 weeks to 13 at 13 weeks) [23], and only $3 \%$ may still occur thereafter [24]. In a previous prospective study by our group, we showed that when sonographers are instructed to perform a survey of fetal anatomy at the early scan, the detection rate can increase to $45 \%$ [4].

The importance of an early diagnosis is that it allows more time for additional investigations, repeat US examinations, and informed parental decision making regarding the course of pregnancy. If termination of pregnancy is chosen, this can occur without time constraint and with less psychological and medical sequelae than at a later stage $[25,26]$. This is also the main reason for women to prefer early screening [27]. Indeed, the relatively high percentage of termination of pregnancy after early detection of anomalies in this study is explained by their severity. Early diagnosis becomes especially important with the introduction of prenatal wide exome sequencing [28, 29]. In case of anomalies with normal array comparative genomic hybridization, there is still enough time to carry out this advanced genetic investigation within the legal term for termination in most countries.

To conclude, in view of the strong decline in FTS after the introduction of cffDNA as first-tier screening for aneuploidies, we recommend the introduction of a 12-13week scan as first part of the routine anomaly screening in pregnancy, as this enables early identification of severe anomalies. The screening can then be completed at around 20 weeks and target especially the more challenging organs, such as the brain and heart. We anticipate that additional training of sonographers and use of the vaginal route when visualization is suboptimal will significantly increase the number of anomalies diagnosed at the 12 13-week scan. 


\section{Statement of Ethics}

This study was approved by the Medical Ethics Committee of the University Hospital.

\section{Disclosure Statement}

The authors report no conflict of interest.

\section{References}

1 Palomaki GE, Haddow JE, Beauregard LJ: Prenatal screening for Down's syndrome in Maine, 1980 to 1993. N Engl J Med 1996;334: 1409-1410.

2 Syngelaki A, Chelemen T, Dagklis T, Allan L, Nicolaides KH: Challenges in the diagnosis of fetal non-chromosomal abnormalities at 1113 weeks. Prenat Diagn 2011;31:90-102.

3 Grande M, Arigita M, Borobio V, Jimenez JM, Fernandez S, Borrell A: First-trimester detection of structural abnormalities and the role of aneuploidy markers. Ultrasound Obstet Gynecol 2012;39:157-163.

4 Kenkhuis MJA, Bakker M, Bardi F, Fontanella $\mathrm{F}$, Bakker MK, Fleurke-Rozema $\mathrm{H}$, et al: Yield of a 12-13 week scan for the early diagnosis of fetal congenital anomalies in the cellfree DNA era. Ultrasound Obstet Gynecol 2018;51:463-469.

5 Czeizel A: Definition of multiple congenital abnormalities. Acta Morphol Acad Sci Hung 1981;29:251-258.

6 Karim JN, Roberts NW, Salomon LJ, Papageorghiou AT: Systematic review of first-trimester ultrasound screening for detection of fetal structural anomalies and factors that affect screening performance. Ultrasound $\mathrm{Ob}-$ stet Gynecol 2017;50:429-441.

7 Iliescu D, Tudorache S, Comanescu A, Antsaklis P, Cotarcea S, Novac L, et al: Improved detection rate of structural abnormalities in the first trimester using an extended examination protocol. Ultrasound Obstet Gynecol 2013;42:300-309.

8 Becker R, Wegner RD: Detailed screening for fetal anomalies and cardiac defects at the 1113 -week scan. Ultrasound Obstet Gynecol 2006;27:613-618.

9 Hildebrand E, Selbing A, Blomberg M: Comparison of first and second trimester ultrasound screening for fetal anomalies in the southeast region of Sweden. Acta Obstet Gynecol Scand 2010;89:1412-1419.
10 Nakling J, Backe B: Routine ultrasound screening and detection of congenital anomalies outside a university setting. Acta Obstet Gynecol Scand 2005;84:1042-1048.

11 Sinkovskaya ES, Chaoui R, Karl K, Andreeva E, Zhuchenko L, Abuhamad AZ: Fetal cardiac axis and congenital heart defects in early gestation. Obstet Gynecol 2015;125:453-460.

12 Hyett J, Perdu M, Sharland G, Snijders R, Nicolaides KH: Using fetal nuchal translucency to screen for major congenital cardiac defects at 10-14 weeks of gestation: population based cohort study. BMJ 1999;318:81-85.

13 Pereira S, Ganapathy R, Syngelaki A, Maiz N, Nicolaides KH: Contribution of fetal tricuspid regurgitation in first-trimester screening for major cardiac defects. Obstet Gynecol 2011;117:1384-1391.

14 Chaoui R, Benoit B, Mitkowska-Wozniak H, Heling KS, Nicolaides KH: Assessment of intracranial translucency (IT) in the detection of spina bifida at the 11-13-week scan. Ultrasound Obstet Gynecol 2009;34:249-252.

15 Bakker M, Pace M, de Jong-Pleij E, Birnie E, Kagan KO, Bilardo CM: Prenasal thickness, prefrontal space ratio and other facial profile markers in first-trimester fetuses with aneuploidies, cleft palate, and micrognathia. Fetal Diagn Ther 2018;43:231-240.

16 Borruto F, Comparetto C, Acanfora L, Bertini G, Rubaltelli FF: Role of ultrasound evaluation of nuchal translucency in prenatal diagnosis. Clin Exp Obstet Gynecol 2002;29:235-241.

17 Taipale P, Ämmälä M, Salonen R, Hiilesmaa $\mathrm{V}$ : Learning curve in ultrasonographic screening for selected fetal structural anomalies in early pregnancy. Obstet Gynecol 2003;101: 273-278.

18 Luchi C, Schifano M, Sacchini C, Nanini C, Sceusa F, Capriello P, et al: Detailed fetal anatomy assessment in the first trimester at 11,12 and 13 weeks of gestation. J Matern Fetal Neonatal Med 2012;25:675-678.

19 Best S, Wou K, Vora N, van der Veyver IB, Wapner R, Chitty LS: Promises, pitfalls and practicalities of prenatal whole exome sequencing. Prenat Diagn 2018;38:10-19.
20 Krakow D, Lachman RS, Rimoin DL: Guidelines for the prenatal diagnosis of fetal skeletal dysplasias. Genet Med 2009;11:127-133.

21 Blaas HG, Eik-Nes SH: Sonoembryology and early prenatal diagnosis of neural anomalies. Prenat Diagn 2009;29:312-325.

22 Souka AP, Pilalis A, Kavalakis Y, Kosmas Y, Antsaklis P, Antsaklis A: Assessment of fetal anatomy at the 11-14-week ultrasound examination. Ultrasound Obstet Gynecol 2004;24: 730-734.

23 Regan L, Rai R: Epidemiology and the medical causes of miscarriage. Baillieres Best Pract Res Clin Obstet Gynaecol 2000;14:839-854.

24 Wilson RD, Kendrick V, Wittmann BK, McGillivray B: Spontaneous abortion and pregnancy outcome after normal first-trimester ultrasound examination. Obstet Gynecol 1986;67:352-355.

25 Korenromp MJ, Page-Christiaens GC, van den Bout J, Mulder EJ, Visser GH: Adjustment to termination of pregnancy for fetal anomaly: a longitudinal study in women at 4 , 8, and 16 months. Am J Obstet Gynecol 2009; 201:160.e1-e7.

26 Kara F, Dogan NU, Bati S, Demir S, Durduran Y, Celik C: Early surgical abortion: safe and effective. Eur J Contracept Reprod Health Care 2013;18:120-126.

27 Maiz N, Burgos J, Barbazán MJ, Recio V, Martínez-Astorquiza T: Maternal attitude towards first trimester screening for fetal abnormalities. Prenat Diagn 2016;36:449-455.

28 Hillman SC, Willams D, Carss KJ, McMullan DJ, Hurles ME, Kilby MD: Prenatal exome sequencing for fetuses with structural abnormalities: the next step. Ultrasound Obstet Gynecol 2015;45:4-9.

29 Vora NL, Powell B, Brandt A, Strande N, Hardisty E, Gilmore K, et al: Prenatal exome sequencing in anomalous fetuses: new opportunities and challenges. Genet Med 2017;19: 1207-1216. 